\title{
Unconstrained Measurement of Heart Rate Considering Harmonics of a Respiratory Signal by Flexible Tactile Sensor Sheet
}

\author{
Kazuya Matsuo \\ Department of Mechanical and Control Engineering, Kyushu Institute of \\ Technology, 1-1, Sensui, Tobata, Kitakyushu, Fukuoka, 804-8550, JAPAN \\ Toshiharu Mukai \\ Meijo University, JAPAN \\ Shijie Guo \\ Hebei University of Technology, CHINA \\ E-mail:matsuo@cntl.kyutech.ac.jp
}

\begin{abstract}
This study describes a heart rate measurement method by considering the harmonics of a respiratory signal. Respiration and heart rates were measured by applying frequency analysis to the time-series data of body pressure. The harmonics of a respiratory signal serve as noises in heart rate measurement. Therefore, heart rate measurement was improved by eliminating the effects of the harmonics. The results of experiments demonstrated that our proposed method enhances the precision of heart rate measurement.
\end{abstract}

Keywords: Harmonics of a respiratory signal, Heart rate measurement, Unconstrained measurement, Sleeping state.

\section{Introduction}

In Japan, the percentage of the elderly, aged 65 or older, against the total population will increase to $29 \%$ in $2020,32 \%$ in 2030 , and surpass $36 \%$ in 2040 [1], [2]. The population of the elderly was estimated to be $2,924,000$ in 2010 and is projected to be $3,619,000$ in 2020 [3]. It is also estimated that the number of individuals requiring care will increase. However, the increase in the number of caregivers may not keep pace with this rise. Therefore, Japan may face an acute lack of caregivers in the immediate future, and the burden on nursing staff is expected to increase. The need for the introduction of a nursing-care apparatus has thus been heightened, and the watching of the perception sickness has been decided by the national government as one of the priority fields.

If the condition of the patient could be assessed using sensors, it could greatly contribute to the reduction of burden on the nursing staff. Conditions of the nursed person to be distinguished include whether they are on the bed and whether their sleeping posture is supine or prone. Addition- ally, measurements of the sleeping state, such as the depth of sleep, would be useful to monitor the health of the nursed person.

Polysomnography (PSG) [4] is a well-known method of measuring the sleeping state. PSG can also diagnose sleep apnea syndrome and periodic limb movement disorder. To recognize the sleeping state, PSG requires biophysiological information, such as brain activity

(C) The 2021 International Conference on Artificial Life and Robotics (ICAROB2021), January 21 to 24, 2021 
(electroencephalograms), heart activity (electrocardiograms), eye movements, muscle activity (electromyograms) of the chin, and respiratory air- flow. The monitored body functions are used to score each period of sleep according to one of the six sleep stages based on the R\&K manual [5]; these include awake, REM sleep, and four stages of non-REM sleep. As a medical specialist makes the final diagnosis, PSG is capable of accurately determining the sleep stage. However, attaching many sensors to the body to obtain numerous biological signals is necessary. Thus, PSG hinders natural sleeping, accompanied by a feeling of restrain. Further, there is a need for the judgment of an inspection technician to distinguish the sleeping state from the biological signal, and thus, automation of this task is difficult. Therefore, a technique that can conveniently estimate the sleeping state is required. The sleeping state can be estimated from biological information such as the respiration rate, heart rate, body motion, and lying posture [6]-[13], which can be measured relatively easily. For example, there is a strong correlation between heart rate and sleep state [6]. Many unconstrained and non- invasive sleep estimation methods have been reported [7]-[11]. Watanabe et al. developed a noncontact algorithm for the sleeping state estimation using heartbeat and body- movement signals [7]. Kambayashi et al. estimated the sleeping state cycle using body-movement density, measured from a passive infrared type motion sensor [8]. Okada et al. proposed a non-restrictive and non-invasive sleeping state evaluation technique from body movements obtained from video images [9]. Deguchi et al. evaluated heart rate variability and respiratory variability [10]. Harper et al. evaluated the potential of the classification of sleep states using cardiorespiratory measures [11]. This paper proposes a method to measure sleep posture [12], respiration, and heart rate [13] using a flexible tactile sensor sheet.

Conventional sleep estimation methods are often basedon the assumption that respiration and heart rates can be accurately measured. However, it is not always possible to accurately measure biological information. In fact, in the method used in [13], the percentage of successful heart rate measurement in overnight experiments was only $40 \%$.

There are several possible reasons for the failure of ac- curate heart rate measurement. For example, if the pressure information corresponding to the type of sleeping posture is used, and if the identification of the sleeping posture fails, accurate measurements of the respiration and heart rates also fail. Additionally, there is a problem that the harmonics of the respiratory signal behave like noise signals in the heart rate measurement.

As the heartbeat signal is weaker than the respiratory signal, the intensity of the frequency spectrum may be smaller than the harmonics of the respiratory signal. In such a case, the heart rate measurement will fail. In this paper, we propose two methods to improve the measurement accuracy of the heart rate by eliminating the effects of the harmonics of the respiratory signal.

\section{Heart Rate Measurement Method Considering Harmonics of a Respiratory Signal}

\subsection{Measurement method of respiratory and heart rates from body pressure}

A method for measuring respiration and heart rates from body pressure information using a flexible planar tactile sensor has been described (Fig. 1) [13]. The body pressure of a person lying on a bed is measured using a tactile sensor sheet. It is observed that the respiration and heart rates remain nearly constant during rest. The frequency bands of respiratory and heart rates do not overlap. Frequency analysis is applied to the time-series data of body pressure, measured by sensor cells in the appropriate position, such as on the chest. A fast Fourier transform (FFT [14], has been used for frequency analysis. Finally, the respiratory and heart rates were acquired by extracting the appropriate frequency band. The frequency bands of the extracted respiratory and heart rate data were determined by preliminary experiments in the following manner: (a) The frequency band of 


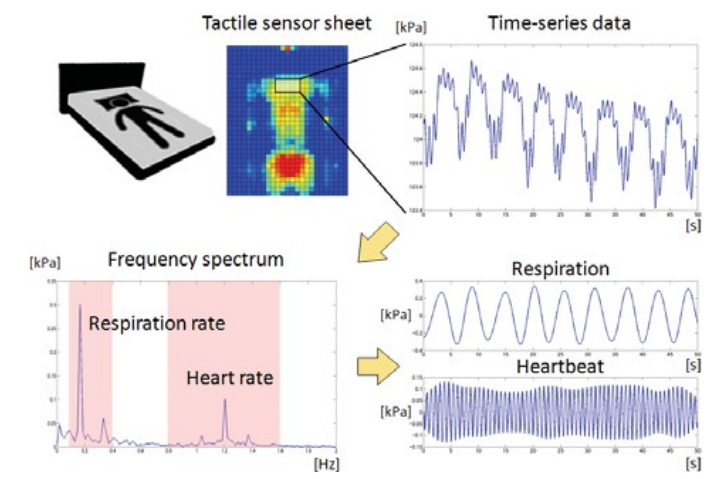

Fig. 1. Measurement method of respiration and heart rates using a flexible planar tactile sensor.

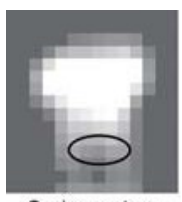

Supine posture

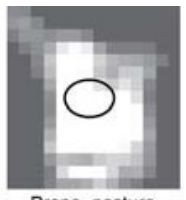

Prone posture
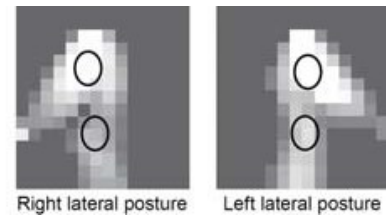

Fig. 2. Appropriate positions for measuring heart rate. The oval shapes show the appropriate positions [12].

respiration is 0.1 to $0.4 \mathrm{~Hz}$ (6 to 24 times/min). (b) The frequency band of the heart rate is 0.8 to $1.6 \mathrm{~Hz}$ (48 to 96 times/min). When obtaining the frequencies of the respiration and heart rates, their dominant frequencies can be found.

Measuring body pressure at appropriate positions such as the chest to estimate the heart rate is necessary. Fig. 2 shows the appropriate positions for heart rate measurement determined by Mukai [12].

\subsection{Heart rate measurement by eliminating respiratory harmonics}

In the conventional method [13], the harmonics of the respiratory signal act as noise signals in the measurement of the heart rate. When the heartbeat signal is weak, the intensity of the frequency spectrum may be smaller than the harmonics of the respiratory signal. Hence, the conventional method sometimes fails to measure the heart rate. In this paper, we propose two methods to improve the measurement accuracy of the heart rate by eliminating the harmonics of the respiratory signal. A sample measurement response of the proposed methods is shown in Fig. 3.

The specifications of the proposed methods to eliminate the harmonics of the respiration are described below.

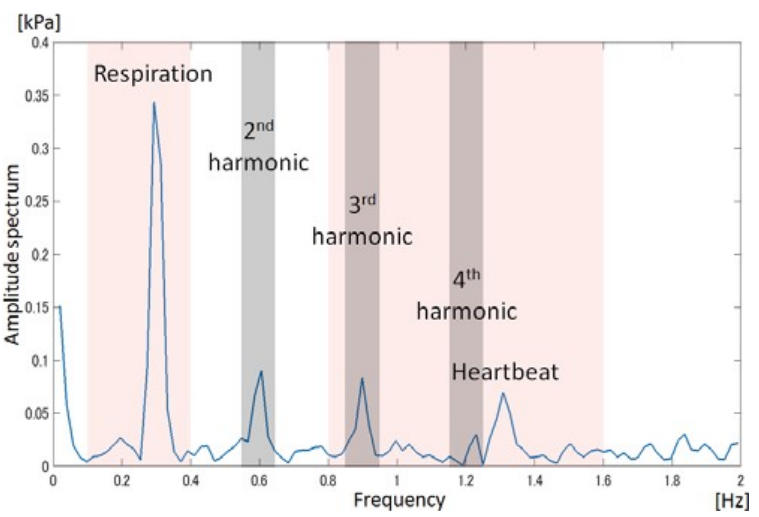

Proposed Method 1: The frequencies of the second, third, and fourth harmonics are calculated from the

Fig. 3. Measurement of heart rate by eliminating respiratory harmonics.

frequency of respiration, obtained using the conventional method. The spectral intensity is then set in the ranges of zero to $0.05 \mathrm{~Hz}$ around the harmonics. The frequency of the heart rate is obtained by finding the dominant frequency in this modified frequency spectrum. The eliminated ranges are determined on the basis of the results of the preliminary experiments.

Proposed Method 2: In the case that a harmonic of the respiratory signal and the frequency of the heart rate are close to each other, proposed method 1 also eliminates the heart rate signal. Thus, some ratio of the intensity of the fundamental respiratory signal is subtracted from those ranges instead of setting the intensity in the ranges to zero. Specifically, $20 \%$ of the respiratory signal is subtracted from the range of the second harmonic and $10 \%$ of the respiratory signal is subtracted from the ranges of the third and the fourth harmonics. These ratios $(20 \%, 10 \%)$ are determined using preliminary experiments.

These two methods are compared with the conventional method to investigate the validity of our proposed methods.

\section{Flexible Tactile Sensor Sheet}

To conduct experiments according to the proposed methods, a sensor that can measure the body pressure of a person lying on a bed at a high resolution is required. To measure the small pressure fluctuation of the heartbeat signal, a high- pressure resolution sensor is required. In addition, to identify the type of lying posture and to measure the body pressure at appropriate positions, a high spatial resolution sensor is required. A Smart Rubber (SR) sensor [15] (Sumitomo Riko, Ltd.), which is a flexible and stretchable planar tactile sensor, made of rubber-based 
materials, including the wiring, was used. Hence, it was sufficiently soft and elastic and was able to measure body pressure without causing the subject to

Fig. 4 shows an outline of the structure of the SR sensor comprising two electrode layers made of conductive rubber sheets and a thin dielectric layer made of urethane. The electrode layers have many long narrow belt-like printed electrodes. The dielectric layer is sandwiched between the two electrode layers. The belt-like electrodes in the two electrode layers are orthogonal to each other, and an electric capacitance sensor cell is formed at every intersection of the electrodes. When one layer has $m$ electrodes, and the other layer has $n$ electrodes, $m \times n$ sensor cells are formed as a whole. When the widths of the electrodes were narrowed, high spatial resolution measurement was realized. In contrast, when the widths were widened, a high-pressure resolution measurement was realized.

When a vertical force is exerted on the SR sensor, the dielectric layer is deformed to be thinner. The electrostatic capacitance of the sensor cell increases because the distance between the two electrode layers becomes shorter. The pressure distribution on the SR sensor was calculated by measuring the electrostatic capacitances of all cells. Many pressure sensors having the same mechanism have been proposed. For instance, XSENSOR is commercially available [16]. However, this sensor is expensive and is less stretchable owing to its metal electrodes. It is, thus, not suitable for use in medical and nursing care fields. However, the SR sensor is suitable for these fields. As this sensor is made of rubber printing, it is affordable and stretchable.

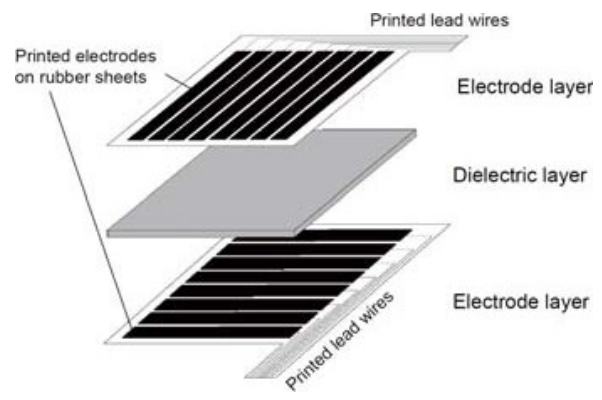

Fig. 4. Outline of the SR sensor feel any discomfort. The body pressure could be measured non-invasively, and without restraint.

structure.

Table 1 summarizes the specifications of the SR sensor used in this study. The size of a sensor cell enables sufficient spatial resolution to distinguish sleep postures. In contrast, when the sensor cell is smaller, the pressure resolution deteriorates. This sensor can measure respiration without any problems because of large pressure fluctuations. However, the heart rate cannot be measured from the noise because the pressure fluctuation is small. Therefore, to measure the heart rate, increasing the pressure resolution by suppressing the noise is critical. The signal-to-noise ratio can be improved by sampling many times, compared to the signal period at high frequency and by obtaining the average of these signals. This technique is called oversampling. In this study, the noise is suppressed by taking an average of 46 samples that were collected.

Table 1. Specifications of the SR

\begin{tabular}{|c|c|c|}
\hline \multicolumn{2}{|c|}{ Sensor } & 800 \\
\hline \multirow{2}{*}{$\begin{array}{l}\text { Number of sensor } \\
\text { cells }\end{array}$} & Length & Width \\
\hline & 32 & 25 \\
\hline $\begin{array}{l}\text { Size of the whole } \\
\text { sensor }\end{array}$ & $\begin{array}{l}882 \\
{[\mathrm{~mm}]}\end{array}$ & $\begin{array}{c}686 \\
{[\mathrm{~mm}]}\end{array}$ \\
\hline S1ze of a sensor cell & {$[\mathrm{mm}]$} & {$\left[\begin{array}{c}14 \\
{[\mathrm{~mm}]}\end{array}\right.$} \\
\hline $\begin{array}{l}\text { S1ze of a gap } \\
\text { between cells }\end{array}$ & $\begin{array}{l}14 \\
{[\mathrm{~mm}]}\end{array}$ & $\begin{array}{c}14 \\
{[\mathrm{~mm}]}\end{array}$ \\
\hline $\begin{array}{l}\text { Thickness of the } \\
\text { sensor }\end{array}$ & \multicolumn{2}{|c|}{$3.5[\mathrm{~mm}]$} \\
\hline $\begin{array}{l}\text { Measuring } \\
\text { frequency }\end{array}$ & \multicolumn{2}{|c|}{$\begin{array}{c}20 \\
{[\mathrm{~Hz}]}\end{array}$} \\
\hline
\end{tabular}

It is possible to measure the heart rate by oversampling. However, it takes a considerable amount of time to oversample all the cells of the sensor; thus, the sampling rate required for measuring the respiration and heart rates cannot be obtained. Therefore, the high-pressure resolution measurement using oversampling should be limited to certain cells. The high-pressure-resolution cells are referred to as the precision cells. In this study, the number of precision cells is four, owing to the limitation of the computational power of the microcomputer mounted on the sensor. As the

(C) The 2021 International Conference on Artificial Life and Robotics (ICAROB2021), January 21 to 24, 2021 
number of precision cells is limited, they have to be appropriately positioned to measure the heart rate.

\section{Experiments}

\subsection{Experimental setup}

To evaluate the effectiveness of the proposed methods, tests were conducted on subjects positioned on the SR sensor and laid on a bed. Eight males aged 21 to 35 years, were selected for this study. This study has been approved by the Research Ethics Committee of the Kyushu Institute of Technology, and an informed consent has been acquired from all the subjects.

The methods were applied to three types of sleeping postures: supine position, prone position, and lateral position. For every posture of each subject, ten measurements were taken. In each measurement, four precision cells were positioned at suitable locations. One hundred and twenty data points per subject were collected. For the FFT, 1,024-point data during $51.2 \mathrm{~s}$ were used $(51.2 \mathrm{~s} \mathrm{x}$ $20 \mathrm{~Hz}=1,024)$.

To calculate errors in the frequencies of the heart rate, the true values of the heart rate were collected using a biological information sensor (biosignalsplux) [17]. The subjects wore the sensor to enable measurements of the true values of the biological information. The sensor measures the subject's heart rate in an electrocardiogram. This sensor also measures respiration by measuring the movement of the chest.

\subsection{Measurement results of heart rate}

Fig. 5 shows the measurement frequency errors of heart rate. The horizontal axis shows the label corresponding to every subject, and the right end indicates the average of all the subjects. The two proposed methods (described in Section II-B) and the conventional method [13] were applied. The vertical axis shows the averages of the absolute values of the errors evaluated by the three methods in the subjects. The error bars show the standard deviations of these values. The average errors of all subjects decreased in the following order: proposed method $2(0.144 \mathrm{~Hz})$, proposed method 1 (0.146 $\mathrm{Hz})$, and the conventional method $(0.149 \mathrm{~Hz})$. The calculation of the paired t-test shows that the difference between the proposed method 2 and the conventional method is statistically significant at the significance level, $p=0.05$ (significance probability: $p=0.011$, t-ratio: $t=2.29)$. In contrast, the calculation of the paired t-test shows that the difference between the proposed method 1 and the conventional method is not statistically significant at the significance level $p=0.05$ (significance probability: $p=0.107$, t-ratio: $t=$ 1.25). The proposed method 2 significantly improves the measurement of the heart rate.

However, the errors of subject-B and subject-G reduce in the following order: the conventional method, proposed method 2, and proposed method 1 , potentially because the harmonics of the respiratory signal and the frequency of the heart rate of these two subjects may be close to each other. In this case, the proposed method 1 eliminates not only the harmonics of the respiratory signal, but also the heart rate signal. Furthermore, even with the proposed method 2, the peak of the heart rate signal is lower, thus increasing the possibility of measurement failure.

Table 2 summarizes the number of data points where the harmonics of the respiratory signal and frequency of the heart rate are close to each other for each subject. Data, where the absolute value of the frequency difference is $0.05 \mathrm{~Hz}$ or less, are considered to be close. Of the 120 data points per subject, the number of data points where the second, third, and fourth harmonics of the respiratory signal and frequency of the heart rate are close to each other is shown in the columns titled 2nd, 3rd, and 4th in Table 2. Subject-B and subject-G have more data where the harmonics of the respiratory signal and frequency of the heart rate are closer to each other than the other subjects. This is believed to be the reason for the measurement error of heart rate, which was greater with the proposed method than with the conventional method for these two subjects. 
Table 2. Date points where the absolute value of the difference between the harmonics of the respiratory signal and the frequency of the heart rate is $0.05 \mathrm{~Hz}$ or less.

\begin{tabular}{|c|c|c|c|c|}
\hline \multirow{2}{*}{$\begin{array}{l}\text { Subject } \\
\text { S } \\
\text { H }\end{array}$} & \multicolumn{3}{|c|}{ Harmonics } & $\begin{array}{l}\text { Number } \\
\text { of } \\
\text { data } \\
\text { points }\end{array}$ \\
\cline { 3 - 4 } A & 0 & 0 & 0 & 0 \\
\hline B & 0 & 4 & 40 & 44 \\
\hline C & 0 & 0 & 8 & 8 \\
\hline D & 0 & 0 & 0 & 0 \\
\hline E & 0 & 0 & 8 & 8 \\
\hline F & 0 & 0 & 0 & 0 \\
\hline G & 0 & 12 & 28 & 40 \\
\hline H & 0 & 0 & 8 & 8 \\
\hline
\end{tabular}

\section{Conclusion}

Two methods were developed to improve the measurement of heart rate by eliminating the effects of the harmonics of the respiratory signal. The experiments showed that the error in heart rate measurement could be reduced by using the proposed method 2 than the conventional method [13]. Our proposed method has enhanced the precision of the heart rate measurement. This will lead to strengthening of measurements of the sleeping state.

For the subjects in whom harmonics of the respiratory signal and frequency of the heart rate were close to each other, the accuracy of measurement of heart rate decreased when the proposed methods were used. The proposed method cannot enhance the precision of the heart rate measurement for such subjects. Identifying such subjects is a future challenge. Additionally, if a parameter for the percentage of the respiratory signal that should be subtracted from the harmonic band in the proposed method 2 can be determined appropriately, this would enable the heart rate to be measured at the same level as the conventional method, even for such subjects. In future research, a method to determine the optimal parameter for each subject will be developed. The method will be able to avoid the deterioration of the accuracy of heart rate measurement.

\section{References}

[1] Statistics Bureau, Ministry of Internal Affairs and Communications, Japan. (2011, Oct.). The 2010 Population Census of Japan. Public announcement on 26 Oct. 2011. [Online]. Available: <http://www. stat.go.jp/english/data/kokusei/index.htm>, (accessed on 3rd Dec. 2020).

[2] National Institute of Population and Social Security Research. (2012, Mar.). Population Projections for Japan (January 2012) - 2011 to 2060-. Public announcement on 30 Mar. 2012. [Online]. Available: $\quad<$ http://www.ipss.go.jp/site-ad/index english/ esuikei/gh2401e.asp>, (accessed on 3rd Dec. 2020). 


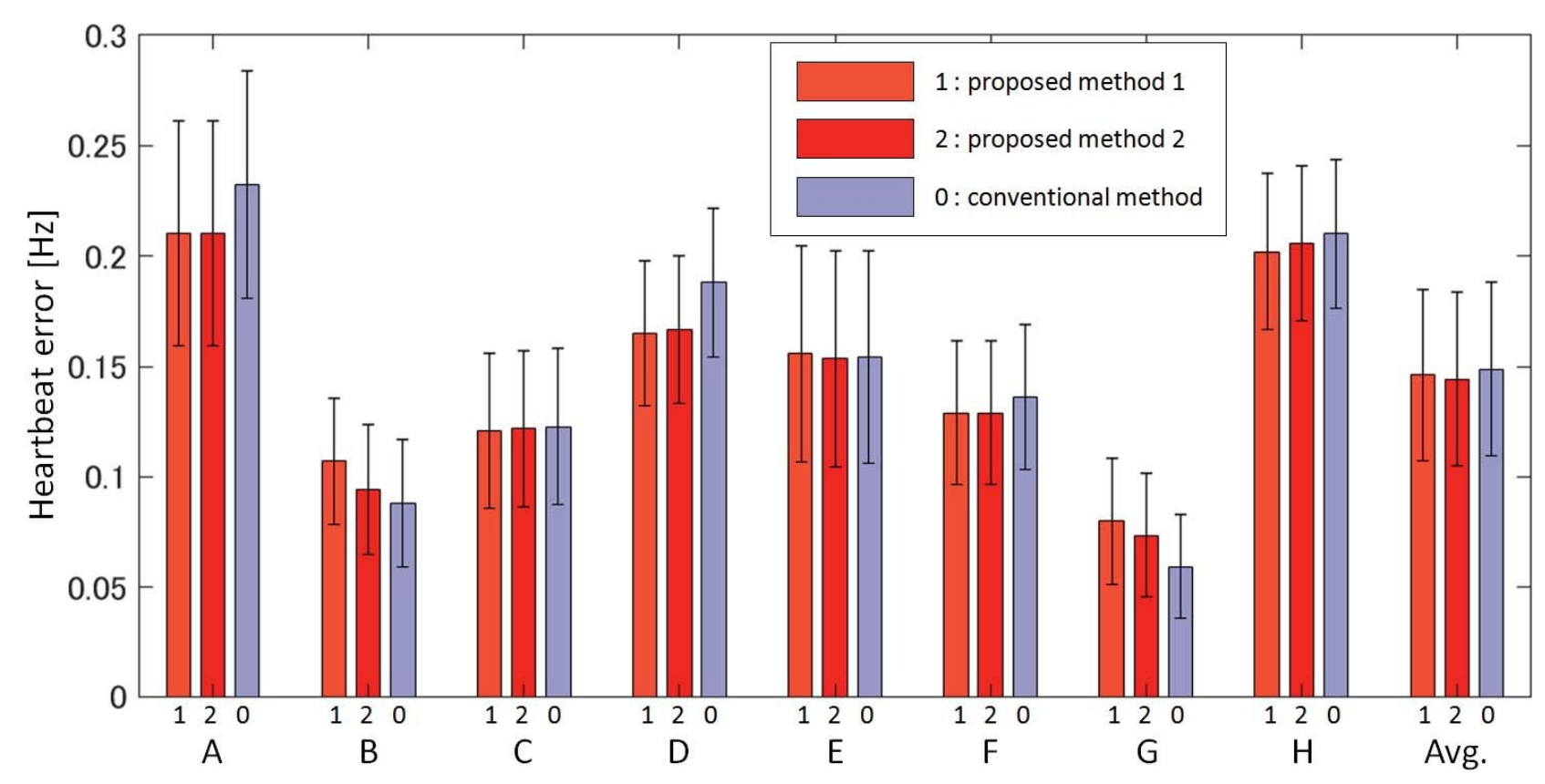

Fig. 7. Measurement frequency errors of heart rate of the two proposed methods and the conventional method. The proposed method 1 eliminates the harmonics of a respiratory signal. The proposed method 2 reduces the effects of the harmonics.

[3] Ministry of Health, Labour and Welfare, Japan. (2011, June). General Welfare and Labour. Public announcement on 2 June 2011. [Online]. Available: $<$ http://www.mhlw.go.jp/english/policy/ other/socialsecurity/index.html>, (accessed on 3rd Dec. 2020).

[4] C. A. Kushida, M. R. Littner, T. Morgenthaler, C. A. Alessi, D. Bailey, J. Coleman Jr, L. Friedman, M. Hirshkowitz, S. Kapen, M. Kramer, T. Lee-Chiong, D. L. Loube, J. Owens, J. P. Pancer, and M. Wise, "Practice parameters for the indications for polysomnography and related procedures: An update for 2005," Journal of Sleep, vol. 28, no. 4, pp. 499-521, 2005.

[5] A. Rechtschaffen and A. Kales, A Manual of Standardized Terminology, Techniques and Scoring System for Sleep Stages of Human Subjects. Bethesda, MD. : U.S. Dept. of Health, Education, and Welfare, Public Health Services-National Institutes of Health, National Institute of Neurological Diseases and Blindness, Neurological Information Network, 1968.

[6] T. Watanabe and K. Watanabe, "Estimation of the Sleep Stages by the Non-restrictive Air Mattress Sensor: Relation between the Change in the Heart Rate and Sleep Stages," Trans. of the Society of Instrument and Control Engineers, vol. 37, no. 9, pp. 821-828, 2001

[7] Y. Kurihara and K. Watanabe, "Sleep-Stage Decision Algorithm by Using Heartbeat and Body-Movement Signals," IEEE Trans. on Systems, Man, and Cybernetics, Part A, vol. 42, no. 6, pp. 1450-1459, 2012.

[8] Y. Kambayashi and H. Hagiwara, "Estimating Sleep Cycle Using Body Movement Density," in Proc. 2012 5th Int'l Conf. on Biomedical Engineering and Informatics, pp. 1029-1033, 2012.

[9] S. Okada, Y. Ohno, Goyahan, K. Kato-Nishimura, I. Mohri, and M. Taniike, "Examination of Non-restrictive and Noninvasive Sleep Evaluation Technique for Children Using Difference Images," in Proc. 30th Annual Int'l Conf. of the IEEE Engineering in Medicine and Biology Society, pp. 2483-2487, 2008

[10] A. Deguchi and H. Hagiwara, "Evaluation of Heart Rate Variability and Respiratory Variability during Sleep Using a
Lorenz Plot," Journal of Sleep and Biological Rhythms, vol. 9, no. 4, p. 366, 2011.

[11] R. M. Harper, V. L. Schechtman, and K. A. Kluge, "Machine Classification of Infant Sleep State Using Cardiorespiratory Measures," Electroencephalography and Clinical Neurophysiology, vol. 67, no. 4, pp. 379-387, 1987.

[12] T. Mukai, K. Matsuo, Y. Kato, A. Shimizu, and S. Guo, "Lying Posture Detection for Unconstrained Measurement of Respiration and Heartbeat of a Person on a Bed," in Proc. 11th IASTED Int'l Conf. on Biomedical Engineering, pp. 46-53, DOI: 10.2316/P.2014.818-014, 2014.

[13] K. Matsuo, T. Mukai, Y. Kato, A. Shimizu, and S. Guo, "Measurement of Respiration and Heartbeat Using a Flexible Tactile Sensor Sheet on a Bed," in Proc. 11th IASTED Int'l Conf. on Biomedical Engineering, pp. 59-64, DOI: 10.2316/P.2014.818-031, 2014

[14] J. W. Cooley and J. W. Tukey, "An Algorithm for the Machine Calculation of Complex Fourier Series," Mathematics of Computation, vol. 19, no. 90, pp. 297-301, 1965.

[15] S. Guo, Y. Kato, H. Ito, and T. Mukai, "Development of Rubber-Based Flexible Sensor Sheet for Care-Related Apparatus," Sumitomo Electric Industries Technical Review, no. 75 , pp. $125-131,2012$.

[16] R. Cork, "XSENSOR Technology: a Pressure Imaging Overview," Sensor Review, vol. 27, no. 1, pp. 24-28, 2007.

[17] PLUX Wireless Biosignals S. A., Biosignalsplux. [Online] Avail- able: <https://plux.info/12-biosignalsplux>, (accessed on 3rd Dec. 2020). 